\title{
¿Favorecen las apps de contactos las conductas de riesgo para las infecciones de transmisión sexual? Análisis de la valoración de personas usuarias según el sexo y la orientación
}

\author{
Naiara Martínez-Gómez \\ al225840@uji.es \\ María Astasio-Centelles \\ al287472@uji.es \\ Cristina Giménez-García \\ gimenezc@uji.es
}

\section{Resumen}

El cambio que los avances tecnológicos han producido en nuestros estilos de vida también parece haber modulado nuestra vivencia de la sexualidad. En este contexto, las apps de contactos podrían facilitar las conductas sexuales de riesgo. Considerando la importancia de analizar la vivencia de las personas usuarias, este estudio busca conocer su percepción del riesgo, así como la valoración que realizan de las propias aplicaciones, según sexo y orientación. Para ello, 93 personas cumplimentaron un cuestionario ad hoc sobre las conductas sexuales online, $54 \%$ mujeres y $46 \%$ hombres; $37,6 \%$ heterosexuales, $32,3 \%$ homosexuales y $29 \%$ bisexuales; con edad media de $30,3$ años (DT $=5,58)$.

Resultados. En cuanto a la valoración del riesgo que implica usar las aplicaciones, en escala de 0 (mucha) a 6 (ninguna), los participantes reportaron una media de $3,58(D T=1,50)$ sin existir diferencias significativas, según sexo u orientación. En cuanto a la valoración de las características que podrían incrementar las conductas sexuales de riesgo, las más identificadas son el anonimato $(35,3 \%)$ y la impulsividad $(26,5 \%)$. A nivel cualitativo, se observa cómo las mujeres otorgan más importancia al anonimato, mientras los hombres la dan a la impulsividad (8,8 \%). En este sentido, heterosexuales y bisexuales identifican en mayor medida el anonimato, mientras los homosexuales la impulsividad. Estos resultados apoyan la necesidad de tener en cuenta dichas características a la hora de elaborar los planes de prevención.

Palabras clave: conductas sexuales de riesgo, nuevas tecnologías (apps), orientación sexual, percepción de riesgo, impulsividad, anonimato. 


\begin{abstract}
The change that technological advances have produced in our lifestyles also seems to have modulated our experience of sexuality. In this context, contact apps could facilitate sexual risk behaviors. Considering the importance of analyzing the experience of the users, this study seeks to know their perception of risk, as well as the assessment they make of their own applications, according to sex and orientation. For this, 93 people completed an ad hoc questionnaire on online sexual behavior, $54 \%$ women and $46 \%$ men; $37.6 \%$ heterosexual, $32.3 \%$ homosexual and $29 \%$ bisexual; with an average age of 30.3 years $(\mathrm{SD}=5.58)$.
\end{abstract}

Results. Regarding the assessment of the risk involved in using the apps, on a scale of 0 (a lot) to 6 (none), the participants reported an average of 3.58 $(S D=1.50)$ without significant differences, according to sex or orientation. Regarding the assessment of its potential to increase risk, the most frequent are anonymity $(35.3 \%)$ and impulsivity $(26.5 \%)$. At a qualitative level, we observe how women attach more importance to anonymity while men give it to impulsivity $(8.8 \%)$. In this sense, heterosexual and bisexual prioritize anonymity, while homosexuals, impulsivity. These results support the need to take these characteristics into account when preparing prevention plans.

Keywords: risky sexual behaviors, new technologies (apps), sexual orientation, risk perception, impulsivity, anonymity.

\title{
Introducción
}

El uso de las redes sociales y las aplicaciones para conocer gente está aumentando en los últimos años; una prueba de ello es el gran número de aplicaciones que surgen con este fin (Hertlein y Blumer 2013; Pichel-Mira 2014; Sabater, Martínez y Campión 2017). Concretamente, las llamadas y los mensajes a través de los teléfonos móviles se han convertido en la forma de relacionarse de las parejas jóvenes (Cortés-Gómez, del Castillo, Hernández y Escobar-Cajamarca 2016; Stokes 2016; Sumter, Vandenbosch y Ligtenberg 2017). En este sentido, supone un mediador en la configuración de las relaciones tanto a nivel intrapersonal como interpersonal (Lasén 2014).

La primera aplicación de citas para móvil (Mobile Dating Apps) surgió en el 2003 y el resto siguió alrededor del 2007. Este tipo de aplicaciones permite lo que se conoce como Satellite dating, momentos transitorios en los que las personas se reúnen a menudo por un solo día o en citas de breve duración (Ayuso y García 2014; Bruno Estefenon y Eisenstein 2015; Slater 2010; Smith y Duggan 2013; Ward 2017).

En la actualidad, la tecnología Smartphone permite el uso de aplicaciones que conectan perfiles de usuario de varias maneras. En ellas, simplemente, los usuarios descargan la aplicación, crean un nombre de perfil, cargan una foto (opcional) y responden a preguntas personales (también opcionales). Más concretamente, algunas aplicaciones permiten notificar cuando las personas que potencialmente pueden atraerles están cerca y conocer si la proporción entre hombres y mujeres se optimiza a su favor (Bruno Estefenon y Eisenstein 2015; Hertlein y Blumer 2013). Además, 
algunos autores defienden que las aplicaciones de citas proporcionan un aumento de la autoestima, así como conocer más información sobre las personas conectadas y poder pensar las cosas antes de dar una respuesta, permitiendo establecer relaciones de mayor confianza y respeto (Fernández, Calvo, Rodríguez y Rodríguez 2018). Estas características convertirían las aplicaciones en un facilitador de prácticas sexuales seguras.

Al mismo tiempo, el uso de estas aplicaciones puede ejercer un papel facilitador como factor de riesgo para la realización de prácticas sexuales inseguras. Tal y como defiende Clark (2015), se establecería una posible relación causal entre el uso de dating apps como Tinder, Grindr y Growlr y el aumento de casos positivos de VIH entre adolescentes. En línea con Cooper, Scherer, Boies y Gordon (1999) y Young (1999), estos medios de comunicación podrían tener algunas características que facilitan la posibilidad de llevar a cabo conductas sexuales de riesgo, como el anonimato, la accesibilidad y la inmediatez. Además, entre las características facilitadoras de prácticas sexuales de riesgo a través de las aplicaciones, cabría enumerar otras como la desinformación (Gil y Romo 2008), la impulsividad (Castro 2010) o la promiscuidad (Baeza, Póo, Vásquez, Muñoz y Vallejos 2007).

Dadas las implicaciones que este fenómeno podría tener para la salud pública y el rol que las propias personas usuarias de aplicaciones pueden jugar, el objetivo de este estudio es valorar su percepción del riesgo para las infecciones de transmisión sexual a través de las aplicaciones, así como la estimación de las mismas en función del sexo y la orientación.

\section{Método}

\section{Participantes}

Participaron 93 personas, de las cuales el $54 \%$ eran mujeres y el $46 \%$ eran hombres. En función de la orientación sexual, el $37,6 \%$ se identificaba como heterosexual; el $32,3 \%$, como homosexual y el $29 \%$, como bisexual; con una edad media de 30,3 años $(\mathrm{DT}=5,58)$.

\section{Instrumento}

Para la investigación se diseñó un cuestionario propio ad hoc, que evalúa la experiencia de uso de las apps de contactos, a través de 59 ítems.

Por una parte, a través de 10 ítems dicotómicos y de escala Likert, se exploran datos sociodemográficos como edad, identidad sexual, orientación sexual, ocupación y situación sentimental. Además, a través de 49 ítems, se evalúa el uso de aplicaciones en las que se conoce a gente a través de un perfil social, como pueden ser Tinder, Happn, Scout, Grindr, Wap@, Hornet, Scruff, Badoo o Adopta un tío. En concreto, se exploran variables como la frecuencia de uso, el número de aplicaciones, el pago de las mismas, la forma de acceso, las imágenes de perfil empleadas, la sinceridad y la finalidad de su uso (motivo de uso, si ha conocido usuarios y si ha mantenido relaciones sexuales); así como áreas en la que ha influido su uso y facilitadores. En este apartado, se incluyen diversos formatos de respuesta: preguntas dicotómicas (9), preguntas de múltiple elección (5), preguntas de elección única (10), preguntas con escala Likert (22) y preguntas de formato de respuesta abierta que permite conocer la opinión de las personas (3). 
En concreto, en el presente estudio se utilizaron las dos preguntas relativas a la percepción de las aplicaciones como factor de riesgo para la salud sexual: 1) una pregunta con escala Likert de 0 (muy de acuerdo) a 6 (muy en desacuerdo), "¿Consideras que las aplicaciones facilitan la realización de conductas sexuales de riesgo para la transmisión de una infección sexual (ITS)?», y 2) una pregunta de formato de respuesta abierta en la que se pedía a las personas que justificaran su valoración, «¿por qué lo consideras?».

\section{Procedimiento}

El presente estudio forma parte de una investigación más amplia sobre la sexualidad en nuevos escenarios, como las aplicaciones de citas. La información relativa al estudio se divulgó mediante las redes sociales, en un mensaje que contenía el enlace al cuestionario online. Una vez los participantes habían dado el consentimiento informado, lo cumplimentaban de forma anónima y voluntaria. Inicialmente participaron 250 personas, pero únicamente cumplieron los criterios de inclusión 93 de ellas, es decir, ser mayor de 18 años y usuario de las apps en la actualidad.

\section{Análisis de datos}

Con el fin de conseguir los objetivos del estudio, se llevaron a cabo dos tipos de análisis. Por un lado, se realizaron estadísticos diferenciales para valorar la posible divergencia de repuesta en función del sexo (prueba t) y la orientación sexual (ANOVA). Por otro lado, para categorizar las respuestas abiertas, se llevó a cabo un análisis de contenido basado en los constructos que la literatura determina como factores facilitadores de las conductas sexuales de riesgo, como son: anonimato, accesibilidad, promiscuidad, impulsividad y desinformación. Una vez categorizadas, se llevó a cabo un análisis diferencial no paramétrico (chi cuadrado) para valorar la divergencia en la frecuencia de aparición de los constructos en función del sexo y la orientación sexual. Los datos se trataron con el programa estadístico SPSS 23.

\section{Resultados}

Los resultados obtenidos como respuesta al ítem del cuestionario « ¿Consideras que las aplicaciones facilitan la realización de conductas sexuales de riesgo?», en una escala 0 (muy de acuerdo) a 6 (muy en desacuerdo), arrojaron puntuaciones medias de 3,58 (DT =1,50), sin existir diferencias significativas entre hombres $(M=3,68$; $\mathrm{DT}=1,61)$ y mujeres $(\mathrm{M}=3,50 ; \mathrm{DT}=1,44)(\mathrm{t}=-0,57 ; \mathrm{p}=0,566)$. En cuanto a la orientación sexual, tampoco se observan diferencias estadísticamente significativas entre heterosexuales $(M=3,71 ; D T=1,48)$, homosexuales $(M=3,33 ; D T=1,60)$ y bisexuales $(M=3,70 ; D T=1,46)(F=-0,60 ; p=0,551)$.

En cuanto a la valoración de las justificaciones, se observa en qué medida las personas participantes mencionan componentes como la inmediatez («Vivimos en la sociedad de la inmediatez, donde todo lo queremos aquí y ahora, y eso afecta también a las relaciones interpersonales de todo tipo»), la instantaneidad («Pueden ser relaciones sexuales más impulsivas e instantáneas») o la falta de control («La mayoría de las veces no se piensa con la cabeza»), que corresponderían al constructo de la impulsividad. A nivel general, se aprecia cómo los hombres y las mujeres homosexuales y los hombres heterosexuales son los que identifican, en mayor 
medida, la impulsividad como un factor facilitador de las prácticas sexuales de riesgo a través de las apps.

Por otro lado, se reporta el desconocimiento sobre la persona («Ya que no se conoce bien a la personas», "El anonimato te permite libertad»), la sinceridad («Hay gente que no es sincera al $100 \%$ y evita comentar esos temas», "Al igual que fuera de la aplicación, arriesgas a no saber nada de su vida sexual anterior, pero sí que es más fácil mentir a través de la aplicación que en persona»), la superficialidad («En mi caso siempre es para un encuentro sexual rápido y esporádico»), la duración («Considero que no se da el suficiente tiempo para conocer a la otra persona y saber si sufre o no alguna enfermedad de transmisión sexual y por ello el riesgo es mucho mayor») y la poca interacción («En mi opinión estas relaciones son de fácil acceso, rápidas y con poca interacción personal entre ambas partes, muy superficiales en todos los sentidos»). Todo lo anterior podría vincularse al constructo del anonimato que, en líneas generales, las mujeres identifican más como facilitador de las prácticas de riesgo.

Por otro lado, las personas usuarias mencionan componentes como la facilidad («Es todo mucho más fácil y rápido y aunque no tiene que ser así es probable que lo sea...», "Creo que las aplicaciones han facilitado el contacto entre personas y siempre habrá personas con conductas tóxicas»), la rapidez («Se busca un sexo rápido y en ocasiones da igual si no hay protección») y el aumento de oportunidades («Creo que las apps te dan accesibilidad y oportunidades»). Estas características podrían entenderse como la accesibilidad, es decir, la existencia de una vía rápida y sencilla para entrar en la aplicación.

Al mismo tiempo, quienes han participado señalan como componentes el número de contactos («Creo que influye la probabilidad de conocer a más gente con la probabilidad de tener más problemas. Pero si se tiene una buena conciencia sexual da igual el resto»), la promiscuidad («La mayoría de estas aplicaciones son usadas para sexo esporádico. La promiscuidad es un factor de riesgo de ETS»), el número de encuentros sexuales («Los encuentros sexuales se acrecientan, con lo cual el riesgo también») y la finalidad del encuentro sexual («La gente solo quiere tener sexo por tener y no miran más allá, por lo que es muy probable que quien tenga alguna ETS no diga nada o tan siquiera lo sepan»). En líneas generales, estas justificaciones podrían incluirse en el concepto de promiscuidad.

Por último, se destacan los componentes de escasa educación sexual («Las infecciones de transmisión sexual son culpa de una mala información en la educación, no por una aplicación de móvil»), la falta de información por parte de los usuarios («Eso depende de cada uno. Tienes que informarte un poco antes. Desde mi punto de vista»), la falta de conocimientos sobre ITS («Si tú tienes las cosas claras, sabes cómo actuar, cómo cuidarte y qué practicas o cuáles no pueden ponerte en riesgo, no tiene por qué haber problema sea cual sea el medio que emplees para conseguir esas relaciones sexuales. Es la persona en sí misma y el conocimiento en profundidad sobre este tema los que permiten protegernos y evitar conductas sexuales de riesgo»), lo que podría asociarse al concepto de desinformación.

A nivel cuantitativo, en lo que se refiere a las características de las aplicaciones relacionadas más frecuentemente con la conducta sexual de riesgo percibido, el anonimato $(35,3 \%)$ y la impulsividad $(26,5 \%)$ serían las más destacables. Tal y como muestra la tabla 1 , las mujeres y los hombres identifican más frecuentemente el anonimato $(37,5 \%$ de mujeres y $30 \%$ de hombres) y la impulsividad $(25 \%$ de mujeres 
y $30 \%$ de hombres). En función de la orientación sexual, las personas heterosexuales y bisexuales identifican con más frecuencia el anonimato como factor facilitador $(42,9 \%)$, mientras que los homosexuales identifican la impulsividad $(38,5 \%)$. No existen diferencias significativas en cuanto a orientación sexual, ni género según el chi cuadrado.

Tabla 1

Análisis diferencial de las características de las apps identificadas como facilitadoras de la conducta sexual de riesgo según sexo y orientación.

\begin{tabular}{|c|c|c|c|c|c|c|c|}
\hline & $\begin{array}{c}\text { Mujer } \\
\%\end{array}$ & $\begin{array}{c}\text { Hombre } \\
\%\end{array}$ & $\begin{array}{l}\mathrm{Chi}^{2} \\
\text { (p) }\end{array}$ & $\begin{array}{c}\text { Heterosexual } \\
\%\end{array}$ & $\begin{array}{c}\text { Bisexual } \\
\%\end{array}$ & $\begin{array}{c}\text { Homosexual } \\
\%\end{array}$ & $\begin{array}{l}\text { Chi }^{2} \\
\text { (p) }\end{array}$ \\
\hline Promiscuidad & 12,5 & 20 & \multirow{5}{*}{$\begin{array}{c}0,52 \\
(0,971)\end{array}$} & 14,3 & 14,3 & 15,4 & \multirow{5}{*}{$\begin{array}{c}4,95 \\
(0,762)\end{array}$} \\
\hline Impulsividad & 25 & 30 & & 14,3 & 28,6 & 38,5 & \\
\hline Desinformación & 12,5 & 10 & & 7,1 & 14,3 & 15,4 & \\
\hline Anonimato & 37,5 & 30 & & 42,9 & 42,9 & 23,1 & \\
\hline Accesibilidad & 12,5 & 12,5 & & 21,4 & 0 & 7,7 & \\
\hline
\end{tabular}

\section{Discusión y conclusiones}

Nuestros hallazgos apoyan pasados estudios que defienden cómo las apps constituyen factores facilitadores de las prácticas de riesgo (Cabecinha et al. 2017; Choi et al. 2016), convirtiendo internet en un elemento modulador de la salud sexual (Beas, Anduaga-Beramendi, Maticorena-Quevedo, Vizcarra-Melgar y Mariño 2016).

Tras los resultados obtenidos, se observa cómo tanto hombres como mujeres atribuyen características similares, viendo que ambos están expuestos al riesgo, tal y como ocurre en otros escenarios (Clark 2015). Además, en función de la orientación sexual, se observa una atribución similar en cuanto a las características de anonimato e impulsividad, tal y como han mostrado pasados estudios (Blackwell, Birnholtz y Abbott 2014; Gómez-Beltrán 2016; Pichel-Mira 2014; Quiroz 2013). Así pues, todos los usuarios de las apps identificarían las mismas características de las apps como facilitadoras de la conducta sexual de riesgo.

En este contexto, cabría tener en cuenta que tanto las expectativas como los objetivos personales podrían influir en el uso y el tipo de conductas que se lleven a cabo, por lo que las variables individuales también serían importantes en el uso de las apps (Stokes 2016). Por ejemplo, Ranzini y Lutz (2017) señalan que las personas usuarias con una alta autoestima tienden a expresar identidades más auténticas y menos engañosas, por ello consideramos que una intervención centrada en el aumento de la autoestima se convertiría en un posible facilitador de prácticas sexuales seguras.

Estos resultados cabría considerarlos en el marco de algunas limitaciones, como el número de participantes, que dificulta generalizar los resultados a otras poblaciones, además del empleo de un autoinforme que podría facilitar la deseabilidad social. Al mismo tiempo, cabría incluir en el análisis otras variables además de la estimación del riesgo, como podría ser la homofobia, la autoestima o la regulación emocional. 
En cualquier caso, el estudio supone una primera aproximación al conocimiento de las apps que confirma su importancia como nuevo escenario para el desarrollo de las relaciones afectivo-sexuales. En concreto, destacaría la necesidad de tener en cuenta las características más notorias, impulsividad y anonimato, a la hora de elaborar los planes de prevención, así como introducir la promoción de la salud sexual en las aplicaciones, dado que el desarrollo de aplicaciones móviles podría favorecer el desarrollo de estrategias de comunicación más cercanas y eficaces.

\section{Referencias bibliográficas}

Ayuso, Luís y Livia García. 2014. Los españoles y la sexualidad en el siglo XXI. Madrid: Centro de Investigaciones Sociológicas.

Baeza, Bernardita, Ana María Póo, Olga Vásquez, Sergio Muñoz y Carlos Vallejos. 2007. «Identificación de factores de riesgo y factores protectores del embarazo en adolescentes de la novena región». Revista Chilena de Obstetricia y Ginecología 72(2): 76-81

Beas, Renato, Alexander Anduaga-Beramendi, Jesus Maticorena-Quevedo, Julissa Vizcarra-Melgar y Christian Mariño. 2016. «Are dating apps a public health issue?». Medwave 16(8): 6536-6536.

Blackwell, Courtney, Jeremy Birnholtz y Charles Abbott. 2014. "Seeing and being seen: Co-situation and impression formation using Grindr, a location-aware gay dating app». New Media \& Society 17(7):1117-1136.

Bruno Estefenon, Susana Graciela y Evelyn Eisenstein. 2015. «La sexualidad en la era digital». Adolescência \& Saúde 12 (2): 83-87.

Cabecinha, Melissa, Catherine H. Mercer, Kirsten Gravningen, Catherine Aicken, Kyle G. Jones, Clare Tanton, Kaye Wellings, Pam Sonnenberg y Nigel Field. 2017. «Finding sexual partners online: Prevalence and associations with sexual behaviour, STI diagnoses and other sexual health outcomes in the British population». Sexually Transmitted Infections 93(8): 572-582.

Castro, Idania 2010. "Conocimientos y factores de riesgo sobre infecciones de transmisión sexual en adolescentes». Revista habanera de ciencias médicas 9(3): 705-716.

Clark, Jocalyn 2015. «Mobile dating apps could be driving HIV epidemic among adolescents in Asia Pacific, report says». BMJ (online) 351. Recuperado de https://www.bmj.com/content/351/bmj.h6493 [Consultado el 4 de agosto de 2019].

Choi, Edmond, Janet Wong, Herman Lo, Wendy Wong, Jasmine Chio y Daniel Fong. 2016. "The association between smartphone dating applications and collage students' casual sex encounters and condom use». Sexual \& reproductive healthcare 9: 38-41.

Cooper, Alvin, Coralie Scherer, Sylvain Boies y Barry L. Gordon. 1999. «Sexuality and the Internet: From sexual exploration to pathological expression». Professional Psychology 30(2): 154-164.

Cortés-Gómez, Ricardo Andrés, Andrea del Castillo, Ángela María Hernández y Manuel Roberto Escobar-Cajamarca. 2016. Configuración de los vínculos erótico-afectivos en las redes sociales: el caso Tinder. Colombia: Pontificia Universidad Javeriana.

Fernández, Diego, Soraya Calvo, Sara Rodríguez y María del Carmen Rodríguez. 2018. "iTienes un Match! Autorrepresentaciones y rasgos comunicativos de las interacciones de jóvenes en Tinder». Revista Mediterránea de Comunicación 9(2): 173-187.

Gil, Eugenia y Nuria Romo. 2008. "Conductas de riesgo en adolescentes urbanos andaluces». Miscelánea comillas 129(66): 493-509. 
Gómez-Beltrán, Iván. 2016. «Resistencias estratégicas a la feminidad masculina en aplicaciones móviles (app) de contacto homosexual entre varones: "Las plumas a otro lado"». Arte y Políticas de identidad 15: 137-154.

Hertlein, Katherine M. y Markie L. C. Blumer. 2013. The Couple and Family Technology Framework: Intimate Relationships in a Digital Age. New York: Routledge.

Lasén, Amparo. 2014. «Remediaciones móviles de subjetividades y sujeciones en relaciones de pareja». En Mediaciones tecnológicas. Cuerpos, afectos y subjetividades, ed. Amparo Lasén y Elena Casado (19-35). Madrid: Universidad Complutense de Madrid y Centro de Investigaciones Sociológicas.

Pichel-Mira, Rafael. 2014. El uso problemático de internet entre los adolescentes. Trabajo de fin de grado. Santiago de Compostela: Universidad de Santiago de Compostela.

Quiroz, Pamela Anne. 2013. «From Finding the Perfect Love Online to Satellite Dating and 'Loving-the- One-You're Near': A Look at Grindr, Skout, Plenty of Fish, Meet Moi, Zoosk and Assisted Serendipity». Humanity \& Society 37(2): 181185.

Ranzini, Giulia y Christoph Lutz. 2017. "Love at first swipe? Explaining Tinder selfpresentation and motives». Mobile Media \& Communication 5(1): 80-101.

Sabater, Carmen, Ion Martínez y Raúl Campión. 2017. «La Tecnosocialidad: El papel de las TIC en las relaciones sociales». Revista Latina de Comunicación Social 72: 1592-1607.

Slater, Bridget. 2010. "Love or Lies: Deception in internet dating». Digital Research \& Publishing 7: 4-13.

Smith, Aaron y Maeve Duggan. 2013. Online dating \& relationships. Pew Research Center: Internet \& Technology. Recuperado de: http://www.pewinternet.org/2013/10/21/online-dating-relationships/ [Consultado el 4 de agosto de 2019].

Stokes, Hannah. 2016. "Come on Baby, Light My Fire: A study on Dutch Millennial Usage of Dating Applications and Morals». Independent Study Project (ISP) Collection: $\quad 2337 . \quad$ Paper Recuperado de: https://digitalcollections.sit.edu/cgi/viewcontent.cgi?referer=https://www.google. com/\&httpsredir=1\&article=3354\&context=isp_collection [Consultado el 4 de agosto de 2019].

Sumter, Sindy, Laura Vandenbosch y Loes Ligtenberg. 2017. «Love me Tinder: Untangling emerging adults' motivations for using the dating application Tinder». Telematics and Informatics 34(1): 67-78.

Young, Kimberly S. 1999. "Internet addiction: Symptoms, evaluation and treatment». Innovations in Clinical Practice 17: 19-31.

Ward, Janelle. 2017. "What are you doing on Tinder? Impression management on a matchmaking mobile app Information». Communication \& Society 20(11): 16441659. 